\title{
Prevalence and Associated Factors of Depression among People Attempting Suicide
}

\author{
Riju Niroula, ${ }^{1}$ Hari Prasad Upadhyay ${ }^{2}$ \\ ${ }^{1}$ Department of Psychiatry, College of Medical Sciences, Bharatpur, Chitwan, Nepal, ${ }^{2}$ Department of \\ Community Medicine, College of Medical Sciences, Bharatpur, Chitwan, Nepal.
}

\begin{abstract}
Background: Suicide is the act of taking one's own life. Suicidal attempts, (both fatal and nonfatal) are a challenging public health issue. Suicide causes heavy morbidity and mortality in the most productive group of a community. This study was aimed to calculate the prevalence rate and associated factors of major depressive disorder among persons who attempted suicide and also to measure the severity of suicidal ideation.
\end{abstract}

\begin{abstract}
Methods: The study involved 50 persons above 18 years of age of both sexes who has attempted suicide in the recent past. An informed consent was obtained from all of them. The prevalence of depression among those suicide attempters was studied based on major ICD- 10 depression inventory. Also, suicide intent rating based on Beck's suicide intent scale was done to assess the severity of suicide attempt. Questionnaire was given and the details were collected.
\end{abstract}

\begin{abstract}
Results: The prevalence of depression is estimated to be $38 \%$ (with $95 \%$ CI $25.86 \%$ to $51.85 \%$ ) among the suicide attempters and $10 \%$ showed high intent for suicide, statistically significant factors are age (pvalue $<0.001$ ), gender ( $\mathrm{p}$-value $=0.002$ ) and suicide intent ( $\mathrm{p}$-value $<0.001$ ).
\end{abstract}

Conclusions: Prevalence of depression is common among people who attempt suicide. Early diagnosis and intervention will reduce suicide attempts.

Keywords: attempted suicide; depression; prevalence; suicidal intent; poisoning.

\section{INTRODUCTION}

Suicide is the act of taking one's own life. Suicidal attempts, (both fatal and nonfatal) are a challenging public health issue. ${ }^{1} \mathrm{~A}$ high suicide rate in any society is an index of social disorganization. ${ }^{2}$ The World Health Organization (WHO) estimates that each year approximately one million people die from suicide, which represents a global mortality rate of 16 people per 100,000 or one death every 40 seconds. In the last 45 years suicide rates have increased by $60 \%$ worldwide. Suicide was the second leading cause of death among 15-29 years olds globally in 2012 It is predicted that by 2020 the rate of death will increase to one every 20 seconds. ${ }^{3}$

Suicide attempts are up to 20 times more frequent than completed suicides. ${ }^{4}$ Although $80 \%$ of persons who commit suicide are men ${ }^{5}$, the majority of those who make nonfatal suicide attempts are women between 25 and 44 years of age. ${ }^{6}$ Thus, suicide causes heavy morbidity and mortality in the most productive group of a community. Regarding methods used in suicide attempts, in most of the cases the attempt was insecticide poisoning.
Majority of the suicide attempters are below the age 30 years. ${ }^{8}$ With regard to mental illnesses causing suicide, depression and other psychiatric illnesses like schizophrenia and personality disorders are commonly associated with suicide attempts, psychosocial issues like lack of social cohesion, unemployment, interpersonal relationship issues were common causes of suicide attempts. ${ }^{8-10}$ Bagadia et al, claim that depression was the most common psychiatric diagnosis made in suicide attempters. ${ }^{11}$ Similar findings have been confirmed in other studies. ${ }^{12,13}$

Suicidal ideation is strongly associated with suicide attempts. In a study by Srivastava and Kumar $17 \%$ of patients with suicidal ideation attempted suicide. ${ }^{4}$ In a study among terminally ill cancer patients, conclude that suicidal ideation and desire for death appeared to be linked exclusively to the presence of a psychiatric disorder. ${ }^{15}$ Among depressed subject's suicidal ideation was present in about two-thirds of the individuals. About $16.6 \%$ of these subjects with suicidal ideation attempted suicide. Attempters also scored high in severity of

Correspondence: Dr. Riju Niroula, Department of Psychiatry, College of Medical Sciences, Bharatpur-10, Chitwan, Nepal. Email: Dr.rijuniroula@gmail.com. Phone: +977-9842029611. Article received: 2019-0824. Article accepted: 2020-03-23. 
Niroula et al. Prevalence and Associated Factors of Depression among People Attempting..

suicidal ideation. ${ }^{16,17}$

Nepal does not have reliable data related to suicide and attempted suicide or prevalence of depression in suicide attempters. The available data are based on police reports or on specific populations, where there is a possibility of gross underestimation. Though Nepal lacks routine national-level data on suicide, or prevalence of depression in suicide, WHO has modelled an age-standardized suicide rate for Nepal in 2012, ranking it 7th in the world at 24.9 per $100000 .^{18,19}$ This reveals that the problem is significant in Nepal. Hence, this study was attempted to estimate the prevalence of depression among persons who attempted suicide and also to measure the severity of suicidal ideation.

\section{METHODS}

A descriptive cross-sectional study was conducted among 50 cases of age $>18$ years who attempted suicide and admitted in College of medical sciences teaching hospital (COMS-TH) Psychiatry ward, were interviewed with focus on psychosocial background, suicide intent rating and depression factors. An informed consent was obtained from all of them. The prevalence of depression among those cases was studied based on major ICD-10 depression inventory. Also, suicide intent rating based on Beck's suicide intent scale was done to assess the severity of suicide attempt. Questionnaire was given and the details were collected. The sample size was calculated on the basis of studies done in India (which has found the overall prevalence of depression among the suicide attempters to be around $20 \%) .{ }^{20}$ By considering this as prevalence, taking $5 \%$ level of significance (The $\mathrm{z}$-score value at $95 \%$ Confidence interval is 1.96), with $10.5 \%$ margin of error, sample size was determined by using the formula, $n=z^{2} p q /$ $\mathrm{e}^{2}=1.96 * 1.96 * 0.2 * 0.80 /(0.11 * 0.11)=50$. Ethical approval was taken from Institutional Review Committee of College of Medical Sciences. At the time of data collection verbal consent with signature was taken first and then data was collected from January 2018 to January 2019. Collected data was checked for completeness and then coded with numbering and then entered in Ms-Excel. Data analysis was done using SPSS-16 software. This is a descriptive study so categorical variables were present in the form of appropriate tables, using frequency (with percentage) and for continuous variables mean and SD were calculate after checking normality of data.

People who had been admitted for attempted suicide and who are above 18 years of age are included in the study. Patients who are critically ill and not willing to participate were excluded from the study. Suicidal intent rating based on Beck's suicide intent scale was done to assess the severity of suicide attempt. Beck's suicide ideation scale- the scale of suicidal ideation consists of 19 items which can be used to evaluate a patient's suicidal intentions. It can also be used to monitor a patient's response to interventions over time and the total score is 38 .

The prevalence of depression among those suicide attempters was studied based on major ICD-10 depression inventory. Major depression inventory (MDI) is a self-report mood questionnaire developed by the World Health Organization which was used in this research. It contains ten items and the scoring pattern is as: mild depression MDI total score of 20 to 24, moderate depression MDI total score of 25 to 29 , severe depression MDI total score of 30 or more.

\section{RESULTS}

Information was collected from 50 patients who attempted suicide. The Mean \pm SD of age was 25.94 \pm 8.77 years. Almost 38 cases $(76 \%)$ of attempted suicide belong to $18-28$ years age group. Among the 50 cases of attempted suicide who were interviewed, 9 are males (18\%) and 41 are females $(82 \%)$. This information was presented in the following (Table 1).

\begin{tabular}{|lcc|}
\hline $\begin{array}{l}\text { Table 1. Age and Gender of Persons } \\
\text { Suicide. }(\mathbf{n = 5 0 )}\end{array}$ & & \\
\hline Category & Frequency & Percentage \\
Age (in years) & 38 & 76 \\
$18-28$ & 5 & 10 \\
$28-38$ & 7 & 14 \\
$38-48$ & $25.94 \pm 8.77$ years \\
Mean(SD) & \multicolumn{2}{|c|}{} \\
Gender & 41 & 82 \\
Female & 9 & 18 \\
Male & & \\
\hline
\end{tabular}

Consumption of insecticides/pesticide (e.g. Organophosphorus compounds) was found in 32 cases $(64 \%)$, corrosives/chemicals (e.g. kerosene, phenols, etc.) was found in 4 cases $(8 \%)$, excess tablet intake (e.g. oral hypoglycemic agents, sleeping pills, etc.) was found in 9 cases $(18 \%)$, and hanging in 5 cases $(10 \%)$ were the common methods chosen. The commonest one being insecticide/pesticide poisoning (Table 2).

\begin{tabular}{|lll|}
\hline Table 2. Methods of suicide attempt. $(\mathbf{n}=\mathbf{5 0})$ \\
\hline Methods of Suicide & Frequency & Percentage \\
Insecticide & 32 & 64 \\
Corrosive & 4 & 8 \\
Medication overdose & 9 & 18 \\
Hanging & 5 & 10 \\
\hline
\end{tabular}

Some attempted suicides are carried out with little to no intention of cessation of life, while others clearly have no other goal. Since it is important to understand a patient's will to die in order to assess the severity of suicide attempt, intent rating was done among the 50 cases of suicide attempt. Of the 
50 cases, $5(10 \%)$ showed high intent, 14 cases $(28 \%)$ showed medium intent and 31 cases $(62 \%)$ showed low intent (Table 3). All 50 cases were

\begin{tabular}{|lll|}
\hline Table 3. Suicide intent among attempters. $\mathbf{n}=\mathbf{5 0}$ \\
\hline Degree of intent & Frequency & Percentage (\%) \\
Low & 31 & 62 \\
Medium & 14 & 28 \\
High & 5 & 10 \\
\hline
\end{tabular}

questioned about how they have been feeling over the last 2 weeks prior to attempt to assess the prevalence of depression among them, 38\% had moderate or severe depression. In that, 5 out of 50 $(10 \%)$ had severe depression, 14 out of $50(28 \%)$ had moderate depression and 31 out of $50(62 \%)$ had no depression, according to MDI, ICD-10 scale (Table 4). The analysis of data revealed that among

\begin{tabular}{|c|c|c|}
\hline Severity of depression & Frequency & Percentage \\
\hline Moderate & 14 & 28 \\
\hline Severe & 5 & 10 \\
\hline No & 31 & 62 \\
\hline
\end{tabular}

50 cases, the prevalence rate of Depression among people who attempted suicide was $38 \%$ (With $95 \%$ CI $25.86 \%$ to $51.85 \%$ ). This information is shown in the (Table 5).The analysis of the data revealed

Table 5. Prevalence of depression among people who attempted suicide. $(\mathbf{n}=\mathbf{5 0})$

\begin{tabular}{lllll|}
\hline Depression & Frequency & Percentage & $\begin{array}{l}\text { 95\% CI } \\
\text { Lower } \\
\text { limit }\end{array}$ & $\begin{array}{l}\text { Upper } \\
\text { Limit }\end{array}$ \\
Yes & 19 & 38 & $25.8 \%$ & $51.8 \%$ \\
No & 31 & 62 & & \\
\hline
\end{tabular}

that depression was seen more in the age group 18-28 years. Among 41 females those who attempted suicide, 19 females were having depression and among 9 males of attempted suicide none had depression. Suicidal intent was found medium to High in 19 cases those who were having depression and suicidal intent was low among all those cases that were not having depression. The statistically significant factors are age ( $p$-value $<0.001)$, gender $(\mathrm{p}$-value $=0.027)$ and suicide intent (p-value $<0.001$ ) (Table 6).

\begin{tabular}{|c|c|c|c|c|}
\hline \multicolumn{5}{|c|}{$\begin{array}{l}\text { Table 6. Association between age, gender and suicidal intent } \\
\text { with depression }(n=50)\end{array}$} \\
\hline Variable & Depression & No Depression & $\begin{array}{l}\text { Chi- } \\
\text { square }\end{array}$ & $P$ value \\
\hline \multicolumn{5}{|l|}{ Age } \\
\hline$<30$ & $8(21.1)$ & $30(78.9)$ & & \\
\hline$\geq 30$ & $11(91.7)$ & $1(8.3)$ & $19.30^{\mathrm{a}}$ & $<0.001^{*}$ \\
\hline \multicolumn{5}{|l|}{ Gender } \\
\hline Female & $19(146.3)$ & $22(57.3)$ & \multirow{2}{*}{$4.904^{\mathrm{b}}$} & \multirow{2}{*}{$0.027^{*}$} \\
\hline Male & - & $9(100)$ & & \\
\hline \multicolumn{5}{|c|}{ Suicidal intent } \\
\hline Low intent & $1(3.1)$ & $31(96.9)$ & & \\
\hline $\begin{array}{l}\text { Medium in } \\
\text { tent }\end{array}$ & $1-13(100)$ & - & $57.50^{\mathrm{c}}$ & $<0.001^{*}$ \\
\hline High intent & $5(100)$ & - & & \\
\hline \multicolumn{2}{|c|}{$\begin{array}{l}{ }^{a} \text { Fisher Exact test value } \\
{ }^{b} \text { Continuity correction } \\
{ }^{c} \text { Likelihood ratio value }\end{array}$} & \multicolumn{3}{|c|}{$\begin{array}{l}\text { * Statistically significant at } 5 \% \text { level of } \\
\text { significance }\end{array}$} \\
\hline
\end{tabular}

\section{DISCUSSION}

This study reveals that among the 50 suicide attempters, $38 \%$ had moderate or severe depression as per ICD-10 major depressive disorder inventory. Depression is commonly associated with suicide attempts. It is commonly under diagnosed. Persons with depression also have high suicide intent. Even in the remaining 31 people who do not fulfill the criteria for depression, many had either one or two symptoms of depression. Several studies indicate that depression is one of the most commonly encountered psychiatric illnesses which often leads to suicidal attempt. ${ }^{11-13,22}$ Hence the need to screen for depression among suicide attempters is essential.

The majority of the suicide attempters (76\%) belonged to 18-28years of age group. This is similar to other Indian studies. Rao, in a study had found that the vulnerable age is from 15 to 25 years. ${ }^{8}$ The particular vulnerability in adolescents and young adults may due to emotional turmoil, interpersonal problems, increase in alcohol and substance abuse, breakdown in extended family, job difficulties and academic setbacks. Thus as large number of this high risk group enters the phase of life associated with greatest risk. This finding highlights the importance of suicide prevention strategies as more number of lives lost in the most productive period of the lifetime.

Out of 50 patients in this study, 41 were females $(82 \%), 9$ were males $(18 \%)$. This shows suicidal attempts were more common in females than males. Similar to findings reported by other studies ${ }^{24}$ where female predominance is noted. Among 41 females those who attempted suicide, 19 females were having depression and among 9 males of attempted suicide none had depression. Females are more likely to ruminate over events than men do, and are more likely than men to become depressed in response to a stressful event. Females are more susceptible to harm from life stressors like interpersonal problem with parents, in-laws, spouse and other family members. These issues can cause feelings of negativity, low self-esteem and lack of control over life in females and are more likely to develop depression with suicidal ideation. This could be probable reason for attempting suicide in these populations. Regarding the methods used in suicide attempts, the most common method of suicide attempt is insecticide poisoning. This finding is similar to other Indian studies. ${ }^{23}$ There has been gradual increase in the rates of poisoning due to easy accessibility of various agents in Nepal. Nandi et al studied the association between easy availability of pesticides and suicide attempts and concluded that it was the motive that determines the suicide attempt and not the easy availability of pesticides. ${ }^{21}$ 
Regarding the suicide intent, of the 50 cases, 5 $(10 \%)$ showed high intent, 14 cases $(28 \%)$ showed medium intent and 31 cases $(62 \%)$ showed low intent. Suicidal intention is strongly associated with suicide attempts. Also suicidal intent was found medium to High in 19 cases those who were having depression and suicidal intent was low among all those cases who were not having depression. Many studies have found that people who exhibit suicide intention are also likely to be depressed and attempt suicide. ${ }^{14-17}$

\section{CONCLUSIONS}

To sum up, the prevalence of depression among people who attempt suicide admitted in psychiatry ward of College of Medical Sciences Teaching
Hospital was estimated to be $38 \%$, among which most of the attempts were by females belonging to the age group below 30years, most commonly by self-poisoning, with medium to high intent. Suicide is a leading cause of mortality among young individuals. Many of them have clinical depression or subclinical symptoms of depression. People expressing suicide intent should be screened for underlying depression. Proper identification, follow up and treatment of depression among suicide attempters are highly essential for successful reduction in suicide mortality.

\section{ACKNOWLEDGEMENTS}

The author would like to thank all the patients for giving consent to participate in the study.

\section{REFERENCES}

1. Bansal P, Gupta A, Kumar R. The Psychopathology and the Socio-demographic Determinants of Attempted Suicide Patients. Journal of Clinical and Diagnostic Research 2011;5(5):917-920.

2. Nagendra Gouda MR, Rao S M. Factors related to attempted suicide in Davanagere. Indian $\mathrm{J}$ of CommunityMedicine.2008;33 (1):15-18.

3. World health statistics 2016: Monitoring health for the SDGs: World Health Organization 2016 World Health Organization. Available at http://apps.who.int/ gho/data/node.main.MHSUICIDE?lang=en retrieved on 26 Aug 2016.

4. Anderson, R. N., Kochanek, K. D., \& Murphy, S. L. (1997). Report of final mortality statistics. Monthly vital statistics report. Hyattsville, Md: National Center for Health Statistics.

5. Moscicki, E. K. Identification of suicide risk factors using epidemiologic studies. PsychiatrClin North Am. 1997; 20:499-517.

6. Fawcett, J., Clark, D. C., \&Busch, K. A. Assessing and treating the patient at risk for suicide. Psychiatr Ann. 1993; 23:244-55.

7. Rao VA. Attempted suicide and suicide among students in Madurai.Indian J Psychiatr. 1972;14:389-97.

8. Rao VA. Attempted suicide.Indian J Psychiatr. 1965;7:253-64.

9. Badrinarayana A. Suicide attempt in Gulbarga. Indian J Psychiatr. 1977;19:69-79.

10. Srivastava MK, Sahoo RN, Ghotekar LH, Dutta S, Danabalan M, Dutta TK, et al. Risk factors associated with attempted suicide. Indian J Psychiatr. 2004;46:33-8.

11. Bagadia VN, Ghadial HN, Shah LP.Unemployment and attempted suicide.Indian J Psychiatr. 1976;18:131-9.

12. Jain V, Singh H, Gupta SC, Kumar S. A study of hopelessness, suicidal intent and depression

in cases of attempted suicide. Indian $\mathrm{J}$ Psychiatr.1999;41:122-30.

13. Badrinarayana A. A study of suicidal risk factors in depressive illness.Indian $\mathbf{J}$ Psychiatr. 1980;22:81-3.

14. Srivatsava AS, Kumar R. Suicidal ideations and attempts in patients with major depression: socio demographic and clinical variables. Indian J Psychiatr. 2005;47:225-8.

15. Latha KS, Bhat SM. Suicidal behavior among terminally ill cancer patients'. Indian J Psychiatr. 2005;45:79-83.

16. Rao AV, Nammalvar N. Death orientation in depression. Indian J Psychiatr. 1979;22:199205.

17. Saxena S, Nepal MK, Mohan D. DSM III Axis diagnoses of Indian psychiatric patients with somatic complaints. AM J Psychiatr. 1988;145:1023-4.

18. Preventing suicide: a global imperative. Geneva: World Health Organization; 2014 (http://apps.who.int/iris/

bitstream/10665/131056/1/9789241564779_e ng.pdf?ua, accessed 19 December 2016).

19. World Health Organization. Global Health Observatory data repository. Suicide rates. Data by country (http://apps.who.int/gho/data/ node.main.MHSUICIDE?lang=en, accessed 3 January 2017).

20. Ramanujam G, Rahuman A, Mahalakshmi R. Prevalence of depression among people who attempt suicide. Int $J$ Res Med Sci 2017;5:4108-11.

21. Nandi DM, Mukherjee SP, Banerjee G, Ghosh A, Boral CG, Chowdry A, et al. Is suicide preventable by restricting the availability of lethal agents? A rural survey of West Bengal. Indian J Psychiatr. 1979;21:251 -5 .

22. Vijayakumar L. Suicide and its prevention: The urgent need in India. Indian J Psychiatr. 2007;49:81-4.

23. National Crime Records Bureau. Suicides in 
Niroula et al. Prevalence and Associated Factors of Depression among People Attempting..

India; 2007. Available at http://ncrb.nic.in/ StatPublications/ADSI/ADSI2007/

Suicides07.pdf. Accessed on 8 July 2017.

24. Beautrais AL, Jyoce PR, Muller RT, Fergusson DM, Deavoll BJ, et al. Prevelence and Comorbidity of Mental Disorders in Persons Making Serious Suicide Attempts; A Case - Control Study. AM J Psychiatr.1996; 153: 1009-1014.

Citation: Niroula R, Upadhyay HP. Prevalence and Associated Factors of Depression among People Attempting Suicide. JCMS Nepal. 2020; 16(1):21-5. 